\title{
Constancy of egocentric visual direction
}

\author{
CYNTHIA LaVERNE MORGAN \\ California State College, Stanislaus, Turlock, California 95380
}

\begin{abstract}
Two experiments investigating the constancy of egocentric visual direction were conducted. In Experiment 1, subjects indicated when a briefly exposed light was subjectively straight ahead during various degrees of asymmetric convergence. The results indicated that the perception of direction exhibited systematic underconstancy. The departures from constancy were dependent upon the degree of asymmetric convergence, increasing as asymmetric convergence increased. Experiment 2 investigated the source of underconstancy found in Experiment 1. The results showed that the underconstancy was due to a combination of the misregistration of eye position and misregistration of the retinal location stimulated. The errors due to misregistration of retinal area were constant regardless of location of the test target, while the errors due to misregistration of eye position were dependent upon the degree of asymmetric convergence. The results were interpreted as supporting a "taking-into-account" model of visual direction.
\end{abstract}

Egocentric visual direction is the perceived location of an object in space with respect to the observer as origin. The egocentric visual direction of an object relative to the median plane of the head can be determined from the position of the eyes in the head and the retinal location stimulated. Since the degree the eyes turn is nearly equivalent to the displacement of an object on the retina, an invariance formulation for perceived direction derived from geometrical considerations takes the form of:

$$
\text { EVD }=\text { REP - ROD, }
$$

where EVD is the perceived egocentric visual direction, REP is registered eye position, and ROD is registered oculocentric direction. The registered oculocentric direction is processed information concerning retinal location that is assumed to be stored or registered in a form which permits it to function as input to the invariance algorithm. Therefore, one can view egocentric visual direction as a simple cancellation process where both registered eye position and retinal location are taken into account and where, when one cancels out the other completely, the egocentric visual direction of an object remains the same (e.g., Epstein, 1973; Shebilske, 1977).

This research was supported in part by Research Grant MH 48463 from the US Public Health Service. The author wishes to thank William Epstein for his invaluable assistance and advice in all phases of this research. This paper is based on part of a dissertation submitted in partial fulfiliment of the requirements for the degree of Doctor of Philosophy from the University of Wisconsin, Madison. Requests for reprints should be sent to Cynthia $L$. Morgan, Department of Psychology, California Siate College, Stanislaus, Turlock, California 95380.
Visual constancies refer to the phenomenon that judged spatial attributes remain more or less invariant despite radical changes in the visual input. A constancy of egocentric visual direction would be inferred if stationary objects are perceived as maintaining an invariant direction from an observer despite changes in the degree of asymmetric convergence that result in the image of the object falling upon different points of the retina.

The geometrical relationship between egocentric visual direction, eye position, and retinal location suggests that in direction perception the ambiguous visual input, viz, the retinal location stimulated, is resolved by taking eye position into account. Similar explanations for other constancies have been advanced (Holway \& Boring, 1941; Kaiser, 1967).

Egocentric visual direction can be understood by assessing the errors due to each component. Hill (1972) provided an experiment that seemed to establish task variations that minimized the possibility of error in first one component of egocentric visual direction and then the other. The procedure used by Hill in his first studies was to have subjects indicate their apparent median plane by setting a light to appear straight ahead during asymmetric convergence. Fixating a light $30^{\circ}$ to one side resulted in a consistent departure from constancy: the apparent median plane shifted approximately $2.5^{\circ}$ in the direction in which the eyes were turned.

Based on the constancy formulation already presented, the evidence from Hill's first studies seemed to indicate that subjects either underestimated the position of their eyes or overestimated the distance from the fovea to the retinal area stimulated. Hill's next studies attempted to deter- 
mine the locus of error. The studies were designed to test the accuracy of each of the two sources of information by minimizing the other source of information in judgments of direction. If eye position information was accurate, subjects should be able to localize accurately (by turning their heads) the direction in which the eyes were turned. If retinal location information was accurate, subjects should be able to localize accurately the direction to an object seen peripherally.

Hill's results indicated an underestimation of the extent necessary to turn the head to the $30^{\circ}$ peripheral target in all conditions. However, in the condition where retinal location information was minimized, the trials were significantly different from the control trials in which the target remained on during head turning. No significant difference between the experimental and control trials was found in the conditions that minimized eye position information. The results were interpreted as indicating a tendency to underestimate the degree to which the eyes were turned after subtracting out the constant error displayed in the control condition. An earlier experiment by Loemker (1930) is consistent with the results and interpretation of Hill's second study.

The literature just cited shows that there is a tendency toward underconstancy in the perception of visual direction and that this may be due to a misregistration of eye position. However, these previous studies have either one or two shortcomings: (1) lack of a wide range of convergence positions, and (2) lack of a control of eye position.

\section{EXPERIMENT 1}

Experiment 1 was designed to investigate the effect of a systematic variation in the degree of asymmetric convergence. The dependent measure was the location of the subject's subjective straight-ahead. In addition to looking at a wider range of eye positions, the present study employed a task which was less susceptible to response bias. The particular task selected was a two alternative forced-choice procedure in which the alternatives were defined by regions of space, viz, the left and right visual field of the observer. Additionally, the study employed at least an indirect procedure for monitoring eye position. Performance on a simultaneous detection task was used to determine if the subject was foveally viewing the fixation target during the presentation of the test flash. Lastly, the present study had a minimal opportunity for posttetanic potentiation (PTP) of the ocular muscles to occur (Paap, 1975). PTP is an involuntary increase in the level of innervation of the ocular muscles induced by prolonged periods of eye turn. Hill's subjects apparently had unlimited time to set the target light to appear straight in front of the head. A shorter uniform time for all subjects to perform the task should reduce any possible effects due to PTP. Shifts in the apparent straightahead reported by Park (1969) can be interpreted as evidence that aftereffects of PTP can play a role in perceived visual direction.

\section{Method}

Subjects. There were six subjects. Two subjects participated for 2077 -trial sessions, while the other four subjects participated in 20121 -trial sessions. All subjects were paid to participate in the experiment.

Apparatus. The same apparatus was used in all the experiments to be reported (see Figure 1). The apparatus was designed to present to the subject an array of lights, equidistant from the midpoint of the line joining the centers of rotation of the subject's eyes (the Cyclopean eye), in a completely dark room. The curvature of the display of lights was such that each light was $50 \mathrm{~cm}$ from the subject's Cyclopean eye. At this viewing distance, each light subtended approximately $.34^{\circ}$ of angle at the Cyclopean eye.

The top row of lights (Row 1), which was at eye level, consisted of 11 light-emitting diodes (LEDs). The 11 LEDs were positioned at $0^{\circ}$ (straight ahead of the subject), $\pm 2^{\circ}, \pm 12^{\circ}$, $\pm 22^{\circ}, \pm 32^{\circ}$, and $\pm 42^{\circ}$ (positive values indicating to the subject's right, negative values indicating to the subject's left) of straight-ahead.

The second row of LEDs (Row 2) was located below the lights just described by $1.75 \mathrm{~cm}\left(2^{\circ}\right)$. This row consisted of 101 LEDs. The distance from the center of one LED to the center of the adjacent LED was $.875 \mathrm{~cm}$, or $1^{\circ}$ at the Cyclopean eye.

The array of LEDs could be manually shifted along the circumference of the equidistance circle so that the center light of Row 1 corresponded to each subject's subjective straight-ahead. This movement could be done to an accuracy of $1 / 2^{\circ}$.

A biteboard and headset arrangement was used to insure that the subject's head was in a normal upright position and to insure a constant head position for each subject throughout the experiment.

General procedure. Each subject was tested individually for a total of $20 \mathrm{~h}$. The subject was seated in a chair in front of the

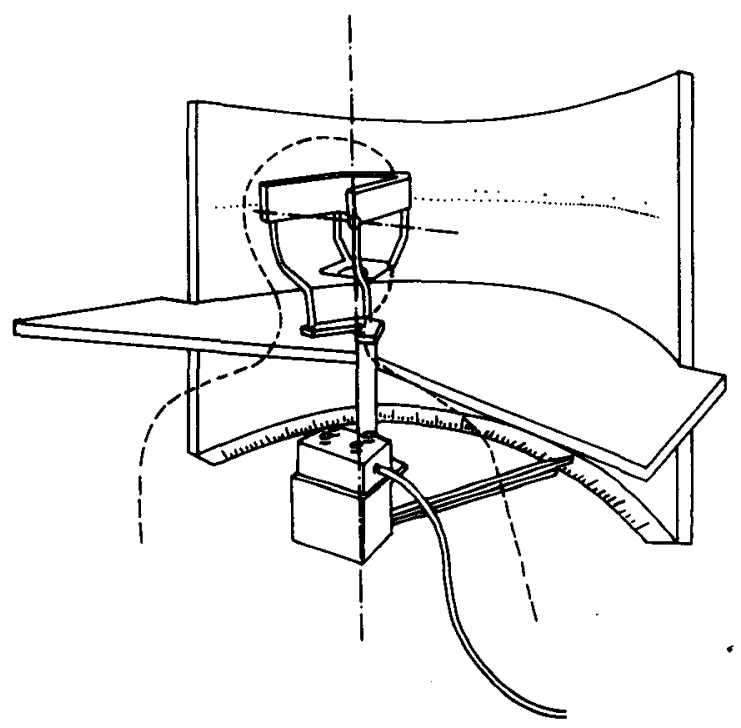

Figure 1. Apparatus. Dashed line indicates subject's head and shoulders. Intersection of dashed-out lines indicates location of Cyclopean eye. Dots on screen represent light-emitting diodes. 
apparatus at a biteboard, in a darkened room. Each test period was approximately $1 \mathrm{~h}$. Binocular vision was used throughout. All testing started after the subject had dark-adapted for approximately $15 \mathrm{~min}$.

Preliminary task. Each LED in Row 1 had a closed ring or a broken ring (Landolt $C$ ) affixed to it. These could be changed by the experimenter periodically. When lit up, the ring appeared black against the red LED. The rings were used as a discrimination task to insure that the subject was fixating a particular light when she was required to.

At the start of each session, a preliminary test determined which size gap in the Landolt $\mathrm{C}$ the subject could discriminate foveally but not $1^{\circ}$ off the fovea. The preliminary test determined the gap size that (1) could be detected above chance when the subject looked directly at the target, but (2) could be detected only at chance level or below when the subject was fixating $1^{\circ}$ right or left of the target.

The purpose of this test was to guarantee that the subject would be fixating a target when she was directed to do so to at least $1^{\circ}$ accuracy. Since fixations which resulted in retinal projections of $1^{\circ}$ off the fovea reduced the identification judgments to a chance level, one could infer that if performance on the identification task in the experiment proper was high, then errors of fixation, if any, were, on the average, less than $1^{\circ}$.

At the completion of this task, each subject's subjective straightahead was found by the method of limits. The subject was asked to indicate which 100 -msec light flash from Row 2 was straight ahead. The back of the apparatus could then be rotated until the center light of the array was moved into the position indicated as the straight-ahead by the subject.

Procedure for practice. After completion of the above task, a series of 11 practice trials on the experimental task occurred. The procedure was as follows: At the ready signal, the subject opened her eyes and looked straight ahead. Shortly after the ready signal, a light from Row I came on. The subject was to move her eyes as rapidly as possible to that light and fixate it. This light had either the closed or the previously determined ring with a gap affixed to it. While still looking at this light, $700 \mathrm{msec}$ later, a 100 -msec test flash from Row 2 occurred at $0^{\circ}, \pm 2^{\circ}, \pm 4^{\circ}, \pm 6^{\circ}$, $\pm 8^{\circ}$, or $\pm 10^{\circ}$ from the subjective straight-ahead. For two subjects out of the six tested in Experiment 1, the 100-msec flash occurred at $0^{\circ}, \pm 2^{\circ}, \pm 4^{\circ}$, or $\pm 6^{\circ}$ from the subjective straightahead. The offset of both lights was simultaneous. The 100 -msec flash was blank. The subject's task was to indicate, by pressing the appropriate buttons, (1) whether the second light appeared to the right or to the left of the straight-ahead, and (2) whether the first light had a closed or an open ring affixed to it. If the subject incorrectly identified the closed or open ring, that trial was repeated later in the session until correctly identified. The practice randomly sampled each of the 11 fixation targets, one test flash per fixation target.

Procedure for experimental trials. Following practice, the experiment commenced. The experimental procedure was identical to that of the practice trials. Instead of 11 trials, there were 77 trials per session (all combinations of the 11 fixation targets and 7 test targets), for 20 sessions, for two subjects and 121 trials per session for the remaining four subjects (all combinations of the 11 fixation targets and 11 test targets). Any incorrect identifications of the closed or open ring were repeated until correctly identified.

The order of fixation targets and test flashes was random, with a new order for each of the 20 sessions. The only restriction for presentation order was that no more than two fixation targets ever occurred on the same side of the subject's subjective straightahead in a row. This was to insure that potentiation would not occur. The intertrial interval averaged approximately $15 \mathrm{sec}$. All sessions were separated, at the minimum, by $1 \mathrm{~h}$.

\section{Results and Discussion}

Preliminary test. All six subjects used a gap width of $1 \mathrm{~mm}$, which subtended a visual angle of $.11^{\circ}$ at the Cyclopean eye. Even though tested at different times and on different days, all subjects used the same gap width during the entire experiment.

The preliminary task results indicated that when fixations resulted in retinal projections of $1^{\circ}$ off the fovea, the identification judgments were reduced to approximately chance.

Subjective straight-ahead. Each subject's subjective straight-ahead did not vary greatly over the 20 sessions. The mean subjective straight-ahead over the 20 sessions was $+1.17^{\circ}(\mathrm{SD}=1.81),+0.77^{\circ}$ $(\mathrm{SD}=2.24),+2.15^{\circ}(\mathrm{SD}=1.71),-3.12^{\circ}(\mathrm{SD}=$ $1.19),-0.75^{\circ}(\mathrm{SD}=1.65)$, and $-3.05^{\circ}(\mathrm{SD}=$ 1.39), for the six subjects (negative values indicating to the left of objective straight-ahead, positive values indicating to the right of objective straightahead).

Identification (gap/circle) errors. All subjects, if errors were made during a session, correctly identified the gap/circle on its second presentation. On the average, no subject made more than 10 errors per session.

Point of subjective equality (PSE). Two analyses of variance with repeated measures were performed. The first analysis was performed on the difference scores (the difference between each subject's PSE at each fixation location and the PSE at $0^{\circ}$ ). A positive sign indicates that the PSE shifted to the subject's right, and a negative sign indicates that the shift was to the subject's left. These signed difference scores provide a test of the hypothesis that the subjective straight-ahead shifts in the direction of eye turn. The hypothesis that the magnitude of the shift is dependent upon the degree to which the eyes are in asymmetric convergence was tested by an analysis of the main effect of location of the fixation target on the absolute value of the difference scores. These two analyses were performed separately on the group data for all six subjects and for the four subjects who had the 121 trials per session.

When the analysis was performed on the signed scores for the six subjects, the main effect of direction of the fixation target was significant, $F(1,5)$ $=556.15, \mathrm{p}<.001$. The mean PSE for fixation targets to the right was $2.64^{\circ}$, while for fixation targets to the left, it was $-3.23^{\circ}$. The main effect of location of the fixation target was not significant. However, the interaction was significant, $F(4,20)$ $=57.60, \mathrm{p}<.001$ (see Figure 2 ). When the analysis was performed on the absolute scores for the six subjects, the main effect of direction of the fixation target was not significant. The main effect of location of the fixation target was significant, $F(4,20)$ $=62.88, \mathrm{p}<.001$ (see Column 2, Table 1). The interaction was not significant.

In the analysis of the signed scores for four subjects, the only different result was that the main 


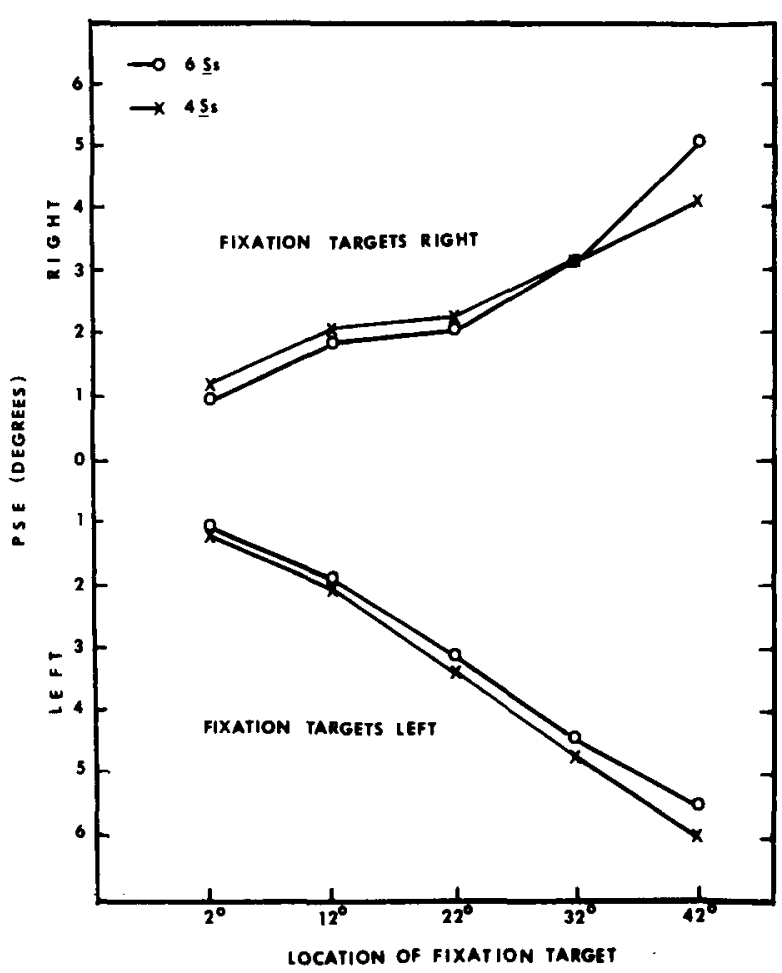

Figure 2. Signed values of the difference scores for six and four subjects and for fixation targets to the right and left.

effect of location of fixation target was significant, $F(4,12)=5.98, p<.01$. In the analysis of absolute scores for the four subjects, the only different result was that the interaction was significant, $F(4,12)$ $=5.52, \mathrm{p}<.01$. The shift in the subjective straightahead was greater for fixations to the left than for fixations to the right.

The results of Experiment 1 clearly indicate that systematic departures from constancy occurred. With the eyes turned to the side, the subjective straight-ahead shifted in the direction of the eye turn. The departures were clearly related to the degree of eye turn, increasing with increasing degrees of asymmetric convergence, whether eye turn was to the right or to the left.

Table 1

Mean Shift in Subjective Straight Ahead (Experiment 1) and Errors in Visual Direction (Experiment 2) (in Degrees)

\begin{tabular}{|c|c|c|c|c|}
\hline \multicolumn{2}{|c|}{ Experiment 1} & \multicolumn{3}{|c|}{ Experiment $2 \dagger$} \\
\hline \multirow{2}{*}{$\begin{array}{c}\text { Target } \\
\text { Location* }\end{array}$} & \multirow{2}{*}{$\begin{array}{l}\text { Obtained } \\
\text { Mean** }\end{array}$} & \multicolumn{2}{|c|}{ Convergence } & \multirow{2}{*}{$\begin{array}{l}\text { Sum of } \\
\text { Errorst }\end{array}$} \\
\hline & & Symmetric & Asymmetric & \\
\hline 2 & 1.09 & & & \\
\hline 12 & 1.89 & 2.48 & -.19 & 2.67 \\
\hline 22 & 2.66 & 2.55 & -1.00 & 3.56 \\
\hline 32 & 3.80 & 2.71 & -1.71 & 4.42 \\
\hline 42 & 5.30 & 2.54 & -2.47 & 5.01 \\
\hline
\end{tabular}

*In degrees. **Absolute values. fUnderestimation (-) and overestimation $(+)$ of target location. H+Symmetric minus asymmetric convergence.

\section{EXPERIMENT 2}

The first experiment indicated that subjects either underestimated the position of the eyes in the head or overestimated the retinal area stimulated by the target. Experiment 2 was designed to test each of these two sources of information when the other source was minimized during judgments of visual direction.

To test the accuracy of the two sources of information, a pointing task was decided upon. A pointing task may produce a systematic bias dependent upon the preferred and utilized hand (Foley \& Held, 1972). Therefore, any effect of the misregistration of eye position information or retinal location information would be exhibited in terms of a difference between trials in which this error would be evident and trials in which only the errors inherent in the pointing task itself would be evident.

Two convergence positions were used in Experiment 2. These positions were designed to test the accuracy of eye-position information and retinal location information when errors in one of the two components were minimized. In the symmetric convergence condition, the subject was instructed to fixate a target located at the subjective straightahead. Changes in the perceived egocentric direction relative to the subjective straight-ahead were determined as a function of changes in the objective position of the test target relative to the subjective straight-ahead. If both registered eye position and registered oculocentric direction are assumed to have a value of zero when the subject fixates the subjective straight-ahead (i.e., when perceived egocentric direction is equal to zero), then the extent to which the obtained perceived egocentric direction departs from the objective position will reflect changes ("errors") in either one component or another, depending upon whether registered eye position or registered oculocentric direction has been maintained at its subjective origin. In the symmetric convergence condition, the subject was instructed to point at a test target located in the peripheral visual field while fixating the subjective straight-ahead. Since registered eye position is at its subjective origin, the obtained perceived egocentric direction reflects the change in registered oculocentric direction induced by the particular retinal-location displacement tested. In the asymmetric convergence condition, the subject was instructed to look directly at a displaced target while she adjusted the pointer. In this case, registered oculocentric direction is at its subjective origin. Consequently, the obtained perceived egocentric direction reflects the change in registered eye position induced by the particular eye turn used.

In the symmetric convergence condition, a $0^{\circ}$ (subjective straight-ahead) fixation target was used to insure that the subject would be in the symmetric 
convergence position while pointing at the peripheral test target. Results of Hill (1972), Matin and Kibler (1966), and Matin, Pearce, Matin, and Kibler (1966) indicate that subjects are unable to maintain their eyes at a previous fixation position without a fixation target present. There was no light present at $0^{\circ}$ in the asymmetric convergence condition, because the presence of two lights might have been a cue for the subject to base her judgments on retinal location information rather than on eye-position information.

\section{Method}

Subjects. There were 24 subjects. All were University of Wisconsin undergraduates participating as a requirement for introductory psychology. Persons who normally wore framed glasses were not selected, but wearers of contact lenses were accepted. To secure these 24 subjects, 32 were tested. Eight were rejected due to failure to meet selection criteria during the practice trials.

Apparatus. The apparatus was the same as that used in Experiment 1 . In this experiment, only the second row of lights was used, and the pointer, represented in Figure 1, was used by the subjects to make responses. The pointer was located under a broad ledge so that it could not be seen by the subject when she was seated at the biteboard and headset arrangement. A protractor mounted at the rear of the apparatus permitted the experimenter to read the location of the pointer to an accuracy of $1 / 2^{\circ}$. The pointer moved freely along this protractor. The second row of lights was at eye level for all subjects.

General procedure. Each subject was tested individually for $90 \mathrm{~min}$ in a darkened room. Binocular vision was utilized throughout. All testing started after the subject had dark-adapted for approximately $15 \mathrm{~min}$.

The subject's task was to move the pointer until it was directly beneath one of the lights in Row 2. Target lights were located at $0^{\circ}, \pm 12^{\circ}, \pm 22^{\circ}, \pm 32^{\circ}$, and $\pm 42^{\circ}$ from the subjective straightahead. Subjects pointed to the $0^{\circ}$ target twice during the experiment and twice just before the initiation of the experimental trials. The two preexperimental pointing responses were used to establish each subject's pointing bias. The misregistration of eye-position information or retinal location information was inferred from the difference between the error obtained with $0^{\circ}$ target and the error obtained with the noncentral targets.

Each target location was pointed to twice, once from a starting position higher than and once from a starting position lower than the numerical position of the target. The starting position was $7^{\circ}$ more or less eccentric than the true target position. These starting positions were chosen because in Experiment 1 no subject deviated from the subjective straight-ahead by more than $7^{\circ}$.

To insure that the subject's eyes were positioned where they were supposed to be during the pointing task, a discrimination task was performed simultaneously with the pointing task. The discrimination task involved the identification of rings with and without gaps flashed randomly in time and space, $1^{\circ}$ to the right or left of the light the subject looked at in the pointing task. The size of the gap was $2 \mathrm{~mm}$, or $.23^{\circ}$ of visual angle at the Cyclopean eye. This gap size could be detected above chance for a target $1^{\circ}$ next to the fixation light, but could be detected only at chance level or below for a target $2^{\circ}$ right or left of the fixation light. This gap size was determined in a preliminary study prior to the commencement of this experiment.

Procedure for practice. Initially, the subject's subjective straight-ahead was found, as in Experiment 1 . Then 25 practice trials were given on a random combination of convergence conditions and location of targets. There were two tasks: a pointing task and a discrimination task. In the pointing task, the subject rested her index finger on the tip of the pointer while the rest of her hand moved the pointer. The subject used the hand that felt most comfortable.

In the symmetrical convergence condition two lights came on simultaneously; one light was straight ahead of the subject, while the second light was to the right or left of this light by various amounts. The subject's task was to look directly at the light straight ahead and move the pointer to the second light, until it was directly under the second light. Both lights were extinguished at the same time. In the asymmetric convergence condition, one light came on, either straight ahead or to the right or left of straight-ahead by various amounts. The subject's task as to turn her eyes directly to this light and also move the pointer to be directly under it. The subject was given $5 \mathrm{sec}$ to complete the pointing response.

As soon as the light(s) went out, the subject was instructed to close her eyes until told to reopen them at the start of the next trial. While the subject's eyes were closed, she was told to keep her eyes in the straight-ahead position. In between trials, the pointer was repositioned so that at the start of each trial the pointer was in a new position.

The discrimination task was performed simultaneously with the pointing task. In the symmetrical convergence condition, briefly displayed lights were periodically flashed, one at a time, around the fixation target located at the subjective straight-ahead. These flashing lights had either a closed ring or a ring with a gap affixed to them. The flashing lights were presented at random times and placed to either side of the fixation light. Discrimination targets were similarly presented around the fixation target in the asymmetrical convergence condition. The subjects were to press the appropriate button (with the hand not used for pointing) to indicate whether the flash contained a ring or a ring with a gap.

If the subject could perform the discrimination task on the practice trials, she went on to the main experiment. Between the practice trials and the experiment itself, the arrangement of the discrimination targets was altered; two trials of pointing to the subjective straight-ahead were given; and then the experiment commenced.

Procedure for experimental trials. There were 34 experimental trials per session (all combinations of the two convergence conditions, two starting positions, and eight target locations, plus two conditions of pointing at the subjective straight-ahead). The procedure was identical to that used in practice, except that any incorrect identifications of the discrimination targets were repeated until correctly identified. Time between trials was approximately $30-45 \mathrm{sec}$.

\section{Results and Discussion}

The average number of discrimination task errors per session was five. The errors were not systematic, indicating that fixation errors, if any, were random.

Pointing bias was assessed by the subject's localization of the subjective straight-ahead. The mean for pointing trials accompanied by the discrimination task did not differ significantly from the mean for pointing trials alone for any subject, indicating that the presence of the discrimination task did not alter the subject's pointing. Constant errors ranged from $-8^{\circ}$ to $+8^{\circ}$. Adjusted scores were determined by subtracting this pointing bias from each obtained pointing response.

A four-factor repeated measures analysis was performed on the adjusted scores: convergence position (symmetric or asymmetric), four target locations, two directions of target location (left or right of subjective straight-ahead), and two pointer starting 
positions (high or low). The main effect of convergence condition was significant, $F(1,23)=65.71$, $\mathrm{p}<.001$. Subjects pointed farther in the symmetric convergence condition than in the asymmetric convergence condition on the average $\left(29.57^{\circ}\right.$ and $25.66^{\circ}$, respectively). The main effect of location of targets was significant, $F(3,69)=63.62, p<.001$. Subjects pointed farther away for the lights that were farther away. Mean adjusted pointing scores were $13.14^{\circ}$, $22.77^{\circ}, 32.50^{\circ}$, and $42.04^{\circ}$ for the $12^{\circ}, 22^{\circ}, 32^{\circ}$, and $42^{\circ}$ targets, respectively. Starting position was also significant, $F(1,23)=12.44, p<.01$, reflecting the difference between the high and low starting positions. The mean for the high starting position was $28.05^{\circ}$, and for the low starting position it was $27.18^{\circ}$. The Convergence Condition by Location of Target interaction was significant, $F(3,69)=4.35$, $\mathrm{p}<.01$. The difference between the asymmetric convergence condition and the symmetric convergence condition increased as the targets became more eccentric (see Column 5, Table 1). The mean adjusted scores were $11.81^{\circ}, 20.99^{\circ}, 30.29^{\circ}$, and $39.53^{\circ}$ for the $12^{\circ}, 22^{\circ}, 32^{\circ}$, and $42^{\circ}$ targets, respectively, in the asymmetric convergence condition, while they were $14.48^{\circ}, 24.55^{\circ}, 34.71^{\circ}$, and $44.54^{\circ}$ for the $12^{\circ}$, $22^{\circ}, 32^{\circ}$, and $42^{\circ}$ targets in the symmetrical convergence condition. Neither the main effect of direction of the targets-right or left of subjective straight-ahead-nor any of the remainder of the interactions was significant.

A four-factor within-subjects analysis of variance was also performed on the differences between the adjusted scores and the true target location, with positive values indicating pointing scores greater than the location of the target and negative values indicating scores less than (short of) the location of the target. The main effects of convergence condition, $F(1,23)=65.71, p<.001$, location of targets, $F(3,69)=2.89, p<.05$, and starting position, $\mathrm{F}(1,23)=12.93, \mathrm{p}<.01$, were significant, as was the Convergence Condition by Location of Target interaction, $F(3,69)=4.35, p<.01$. Neither the main effect of direction of the targets nor any of the remainder of the interactions was significant.

The significant main effect of convergence condition indicated that in the asymmetric convergence condition, subjects consistently pointed short of the target $\left(-1.34^{\circ}\right)$, while in the symmetrical convergence condition, subjects pointed beyond the location of the target $\left(+2.57^{\circ}\right)$. The main effect of starting position indicated that subjects had a small bias in the direction of the starting position. The Conyergence Condition by Location of Target interaction indicated that the effect of target location depended upon the convergence condition. The differences in the symmetric convergence conditions were consistently around $+2.5^{\circ}$ across all four target locations (see Column 3, Table 1). However, for the asymmetric convergence condition, the errors increased with target location (see Column 4, Table 1).

The errors derived from the sum of the errors obtained in Experiment 2 (Column 5, Table 1) were almost identical to the obtained values in Experiment 1 (Column 2, Table 1). Four $t$ tests indicated that for each target location, the differences were not significant.

The results of Experiment 2 can be interpreted as showing both a tendency to underestimate the degree to which the eyes were turned and to overestimate the retinal area stimulated by a peripheral target. When retinal location information is minimized by having the subject use foveal vision only (asymmetric convergence condition), the subject points short of the target. This indicates an underestimation of eye-position information. When eye-position information is minimized by having the subject maintain her eyes straight ahead (symmetric convergence condition), the subject points beyond the target. This indicates an overestimation of retinal location information.

As previously mentioned, Foley and Held (1972) noted a systematic bias dependent upon the preferred and utilized hand. Specifically, they found that when the favored hand and the hand used and the sighting eye are all on the same side, errors in pointing at visual targets without sight of the hand tended to be to the opposite side. All subjects in Experiment 2 used their preferred hands for pointing, which turned out to be the right hand for all subjects, but the biases exhibited ranged from $-8^{\circ}$ to $+8^{\circ}$. This lack of a consistent bias among subjects lessens the possibility that the errors found were simply the result of hand biases. The lack of a systematic bias may be due to the utilization of a sagittal movement of the arm. The biases observed by Foley and Held may be important for a reaching response but not for sagittal arm movements.

\section{GENERAL DISCUSSION}

The results of Experiment 1 showed that there was a departure from constancy of visual direction. The departure from constancy was dependent upon the degree of eye turn, resulting in a monotonic function, increasing as the amount of eye turn increased. The results of Experiment 2, which examined the locus of the error found in Experiment 1, showed that the underconstancy was due to a combination of misregistration of eye position and retinal location, the errors due to misregistration of retinal location being constant regardless of the location of a peripherally seen target, while the errors due to misregistration of eye position were dependent upon the degree of eye 
turn, increasing with increasing eye turn.

If the invariance formulation presented in Equation 1 is correct, the sum of the errors due to eye position and retinal location information should predict the errors in perceived egocentric direction when both components are shifted from their origins. For each target location, the differences between the errors from Experiment 2 and the obtained errors in Experiment 1 were no more than $.9^{\circ}$ (see Table 1).

The small and nonsignificant deviations between these errors at each target location support the contention that the departures from direction constancy found in the first experiment were due to both a misregistration of retinal location information and eye-position information. These experimental results support the "taking-into-account" model of visual direction of Epstein (1973), whereby retinal location information is processed along with extraretinal information that allows eye movements to be taken into account.

Corroborative support for the misregistration of eye position found in Experiment 2 comes from a study dealing with the constancy of object orientation (Ebenholtz \& Paap, 1973). In part of the series of studies by Ebenholtz and Paap, subjects matched the orientation of a horizontal line target with a variable comparison line. The standard line was either straight ahead or displaced $25^{\circ}$ to the left or right. Judgments of perceived visual direction were also made by having the subject fixate and point at the pivot point of the line. The results of the orientation matching task indicated that stimuli on the right appear to be rotated right side away, while those on the left appear to be rotated left side away. Assuming that the visual input salient to orientation is evaluated in terms of the registered degree of eye turn required to fixate the target, this outcome would be consistent with an underestimation of eye position. Overall, the pointing responses did reveal a tendency toward underestimation, as would be expected if registered eye position was being underestimated. However, some groups of subjects viewing lines at various orientations showed no error or a slight tendency toward overestimation. Rank-order correlations between errors in the orientation matching task and in the pointing task tended to be positive, but low and nonsignificant. These inconsistencies might be attributed to (1) the fact that eye position was not rigorously controlled, or (2) the fact that an error of only $1^{\circ}$ would be expected on the basis of the results of Experiment 2 of the present study.

The larger errors in registered eye position obtained at $32^{\circ}$ and $42^{\circ}$ in the present study suggest that if the Ebenholtz and Paap procedure were replicated with more eccentric targets, the correlation between errors in the orientation matching task and the degree of underestimation in the pointing task would be higher. For example, a target with its pivot point displaced $42^{\circ}$ to the right should appear to be less displaced than its objective position, assuming that registered eye position is being underestimated. This, in turn, should cause a frontal plane target to appear to be rotated right side away.

Considering the ease with which the visual system has been shown to adapt to a variety of optical transformations (Epstein, 1967), it is surprising that errors as large as $5^{\circ}$ are obtained with adult observers. For example, one might expect that the systematic relationship between retinal angle and the eye rotation required to bring the target onto the fovea would permit the calibration of the two systems in such a way as to eliminate error. However, Low (1946) observed that the peripheral retina can be assumed to be an unpracticed sensory area. Likewise, observers seldom make judgments in positions of extreme eccentricity; rather, they turn the head so as to minimize the necessary amount of eye turn. It is therefore likely that, given appropriate feedback, performance under the conditions tested in the present study would improve with practice. Since previous observations have shown that peripheral discriminations improve with practice (Johnson \& Leibowitz, 1974; Low, 1946), such an improvement in visual direction with practice would not be unexpected. Lastly, it is possible that observers may be more accurate when presented with targets in the frontal plane than on a curve with all points equidistant from the Cyclopean eye, because of their experience in looking at objects on flat surfaces such as walls (Shebilske, 1977).

\section{REFERENCES}

Ebenholtz, S. M., \& PaAp, K. R. The constancy of object orientation: Compensation for ocular rotation. Perception \& Psychophysics, 1973, 14, 458-470.

EPSTEIN, W. Varieties of perceptual learning. New York: McGraw-Hill, 1967.

Epstein, W. The process of 'taking-into-account' in visual perception. Perception, 1973, 2, 267-285.

FolEY, J. M., \& HELD, R. Visually directed pointing as a function of target distance, direction, and available cues. Perception \& Psychophysics, 1972, 12, 263-268.

Hill, A. L. Direction constancy. Perception \& Psychophysics, $1972,11,175-178$.

Holway, A. H., \& Boring, E. G. Determinants of apparent visual size with distance variant. American Journal of Psychology, 1941, 54, 21-37.

Johnson, C. A., \& Leibowitz, H. W. Practice, refractive error, and feedback as factors influencing peripheral motion thresholds. Perception \& Psychophysics, 1974, 15, 276-280.

KaIser, P. K. Perceived shape and its dependency on perceived slant. Journal of Experimental Psychology, 1967, 75, 345-353.

LOEMKER, K. K. Certain factors determining the accuracy of a response to the direction of a visual object. Journal of Experimental Psychology, 1930, 13, 500-518.

Low, F. N. Some characteristics of peripheral visual performance. 
American Journal of Physiology, 1946, 146, 573-584.

Matin, L., \& KibleR, G. Acuity of visual perception of direction in the dark for various positions of the eye in the orbit. Perceptual and Motor Skills, 1966, 22, 407-420.

Matin, L., Pearce, D., Matin, E., \& Kibler, G. Visual perception of direction in the dark: Roles of local sign, eye movements, and ocular proprioception. Vision Research, $1966,6,453-469$.

PAAP, K. R. Perceptual consequences of post-tetanic potentiation: An alternative explanation for adaptation to wedge prisms. Unpublished doctoral dissertation, University of Wisconsin, 1975.
PARK, J. N. Displacement of apparent straight ahead as an aftereffect of deviation of the eyes from normal position. Perceptual and Motor Skills, 1969, 28, 591-597.

SHEBILSKE, W. Visuomotor coordination in visual direction and position constancies. In W. Epstein (Ed.), Stability and constancy in visual perception: Mechanisms and processes. New York: Wiley, 1977.

(Received for publication April 18, 1977; revision accepted October $31,1977$. 